\title{
A Qualitative Analysis of Implementing EvidenceNOW to Improve Cardiovascular Care
}

\author{
Debora Goetz Goldberg, PhD, MHA, MBA, Sabar Haghighat, MA, \\ Sneha Kavalloor, MD, MS, and Len M. Nichols, PhD
}

Purpose: The Heart of Virginia Health care (HVH) was a regional cooperative under the EvidenceNOW initiative to assist primary care practices in implementing evidence-based cardiovascular care and building capacity for quality improvement. The HVH implementation team included individuals from multiple universities, quality improvement organizations, and consulting firms. The goal of this study was to understand HVH team member viewpoints on the challenges, strengths, and lessons learned in each phase of the project.

Methods: Qualitative methods were used to facilitate reflection on the implementation and dissemination of the EvidenceNOW initiative in Virginia. In-depth interviews were conducted at the end of the project with $22 \mathrm{HVH}$ team members. A nonparticipant, multidisciplinary research team completed thematic analysis of interview transcripts.

Results: Positive attributes of the HVH initiative included diverse team member skills and areas of expertise, a well-received kick-off event, and a comprehensive set of practice improvement resources. Major challenges included recruiting primary care practices, varying types and capabilities of electronic health records, and working with practices at different transformation stages, with different objectives for participating and involvement in other government initiatives.

Conclusions: Study findings provide insights for future dissemination research and implementation of evidence-based practices in primary care. Challenges experienced in project development can result in a domino effect that could change the project timeline, type of practices recruited for study participation, resource allocation, and planned activities for quality improvement. Effectiveness of external quality improvement support may depend on practice engagement, preexisting organizational structures and processes, availability of resources, and length and continuity of practice facilitation. ( $\mathrm{J}$ Am Board Fam Med 2019;32:705-714.)

Keywords: Evidence-Based Medicine, Evidence-Based Practice, Interdisciplinary Research, Organizational Innovation, Primary Health Care, Quality Improvement, Resource Allocation, Virginia

EvidenceNOW: Advancing Heart Health in Primary Care was an initiative funded by the Agency for Health care Research and Quality (AHRQ) to assist small-to-medium-sized primary care prac-

This article was externally peer reviewed.

Submitted 5 March 2019; revised 30 May 2019; accepted 31 May 2019.

From the George Mason University, Department of Health Administration and Policy, Peterson Family Hall, MS IJ3, 4400 University Drive, Fairfax, VA 22030 (DGG, SK); George Mason University, Department of Sociology Fairfax, VA 22030 (SH); George Mason University, Center for Health Policy Research and Ethics Fairfax, VA 22030 (LMN).

Funding: This research was funded by the Agency for Healthcare Research and Quality (R18HS023913).

Conflict of interest: none declared. tices in implementing evidence-based cardiovascular care, building capacity for quality improvement, ${ }^{1}$ and improving clinician satisfaction. The ABCS of Heart Health, which are evidence-based protocols for prevention and treatment of cardiovascular disease, were a key component of the initiative. These protocols involve aspirin use, blood pressure control, cholesterol management, and smoking cessation for high-risk individuals. ${ }^{2}$ An-

Corresponding author: Debora Goldberg, PhD, MHA, MBA, George Mason University, Department of Health Administration and Policy, Peterson Family Hall, MS IJ3, 4400 University Drive, Fairfax, VA 22030 (E-mail: dgoldbe4@gmu.edu). 
Figure 1. Timeline of project phases with team roles. EHR, electronic health records.

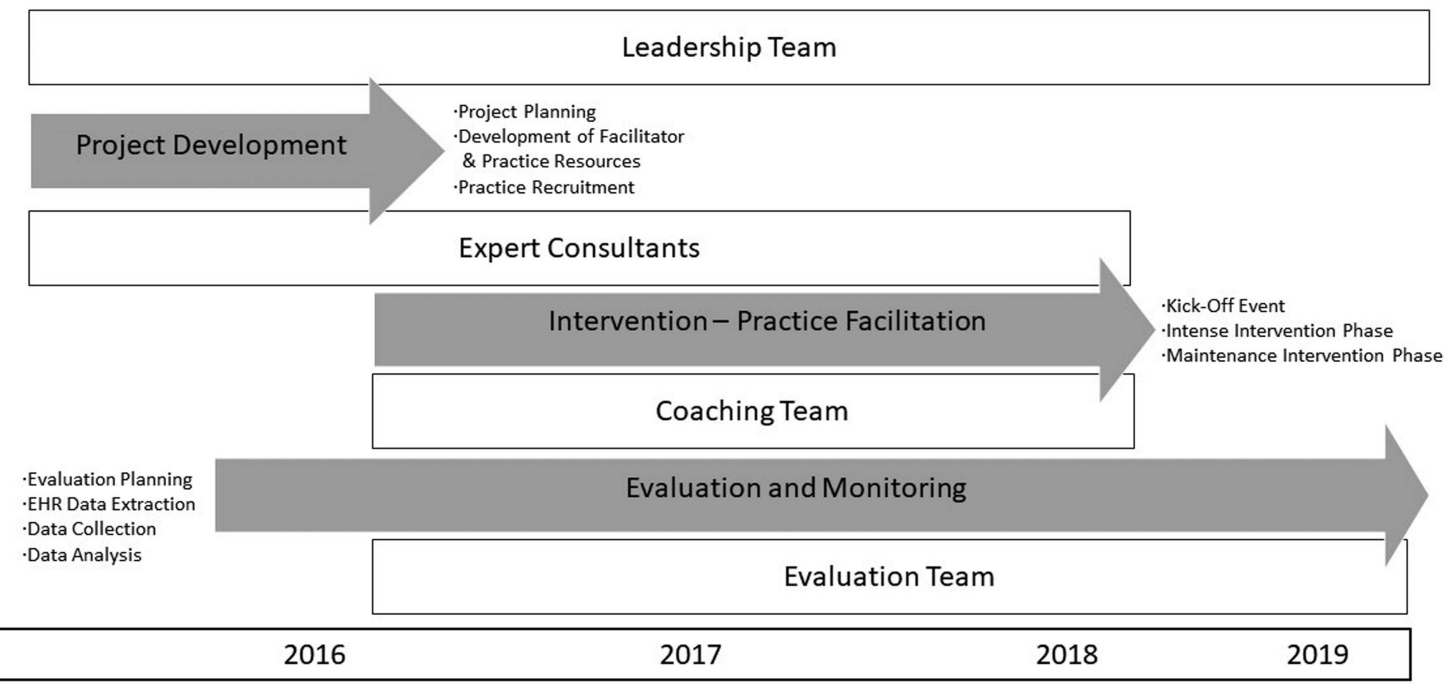

other core component of the EvidenceNOW initiative involved practice transformation through improved use of electronic health records (EHR), data measurement and reporting, coordination of care, and operational efficiency.

Heart of Virginia Health care (HVH) was 1 of 7 regional cooperatives funded under the EvidenceNOW initiative. AHRQ's goals for the regional cooperatives included recruiting 250 primary care practices; incorporating practice coaching and facilitation; using practice data, feedback, and benchmarking; applying peer-to-peer local learning and expert consultation; and conducting a rigorous evaluation that included measurements at baseline, every 3 months during the intervention and 6 months afterward. ${ }^{3}$ Launched in May 2015, regional cooperatives were required to initiate the intervention within 6 months of award and complete quality improvement support within 24 months. ${ }^{4}$ Nevertheless, AHRQ granted time extensions for project development and evaluation activities.

The HVH initiative was a collaborative effort consisting of public and private organizations that had substantial experience in providing support to primary care practices, strong relationships with practices in Virginia, and involvement with similar government initiatives. The cooperative was led by Virginia Commonwealth University and included the Virginia Center for Health Innovation, which provided project leadership and external quality improvement support. Health Quality Innovators, the Virginia Quality Innovation Network-Quality
Improvement Organization, provided practice facilitation on EHR use, population health management tools, and redesign of office processes and reimbursement methods. Community Health Solutions helped in the development of web-based tools and education materials for participating practices. Faculty from George Mason University collected and analyzed practice- and individuallevel data for the evaluation. Several family medicine physicians served as expert consultants to the $\mathrm{HVH}$ team and to participating practices. Most team members were involved throughout the project with varying levels of effort depending on project phase. A timeline of project phases is presented in Figure 1, which illustrates team member involvement.

The Virginia landscape for primary care practices mirrors much of the rest of the United States. Practices are overwhelmed with major changes related to value-based payment methodologies, EHR and International Classification of Diseases, 10th revision (ICD-10) coding implementation, quality measurement and reporting, and coordination of care responsibilities. ${ }^{5,6,7}$ In response, many practices have transitioned to health system ownership ${ }^{8}$ or other organizational and management arrangements.

In Virginia, 220 primary care practices participated in the $\mathrm{HVH}$ initiative. Early findings help portray participating practices, ${ }^{9}$ which included a diversity of practice ownership (32\% independent, $55 \%$ health system owned, and $13 \%$ health center). Typical practices were small, including $71 \%$ with 1 
to 10 physicians; single specialty, $61 \%$; located in medically underserved areas, $69 \%$; and with a high percent of patients, $52 \%$, with publicly funded insurance or uninsured. Practice staff and clinicians indicated, on average, a moderate culture of learning, a moderately positive work environment and psychological safety, and a moderate level of burnout.

In large-scale quality improvement efforts, it is important to reflect on the strengths and weaknesses of each phase in the project's life cycle to help understand key lessons learned for future initiatives. ${ }^{10}$ It is particularly valuable to elicit viewpoints from the implementation team in the dissemination of evidence-based research at the practice level. The purpose of this study was to use reflection as an approach to understand $\mathrm{HVH}$ team member viewpoints on the overall initiative, strengths and weaknesses of the project, and lessons learned.

\section{Methods}

This study was based on implementation and dissemination research that uses qualitative methods involving individual reflection to understand $\mathrm{HVH}$ team members' perceptions of the initiative, experience of working in a collaborative research team, and lessons learned. Reflection is "deliberate thinking about action with a view to its improvement"11 that has been used in previous research to study "beliefs," "experiences," "situation," and "ideas" in medical education and health services delivery. ${ }^{12,13,14,15}$ In this study, we use reflection at the end of the project as an approach to encourage HVH team members to contemplate their experiences and perceptions in each phase of the initiative. The study was approved by the George Mason University Institutional Review Board in September 2017.

\section{Sampling and Recruitment}

We used a population-based sampling method in which all $22 \mathrm{HVH}$ team members were recruited through email and telephone contact for study participation, resulting in a $100 \%$ participation rate. The sample included physicians, academic professionals, project leaders, practice coaches, and expert consultants who participated as part of the HVH implementation and dissemination team; see Table 1 for study participant characteristics. Indi-
Table 1. Characteristics of Study Participants

\begin{tabular}{lr}
\hline Team Member Characteristics $(\mathrm{N}=22)$ & $\mathrm{N}(\%)$ \\
\hline Project role & $7(31.8)$ \\
Leadership team & $10(45.4)$ \\
Coaching team & $3(13.6)$ \\
Expert consulting team & $2(9.0)$ \\
Evaluation team & \\
Professional affiliation & $2(9)$ \\
Virginia Commonwealth University & $3(13.6)$ \\
Virginia Center for Health Innovation & $10(45.4)$ \\
Health Quality Innovators & $2(9.09)$ \\
George Mason University & $2(9.09)$ \\
Community Health Solutions & $3(13.6)$ \\
Independent Consultants & \\
Sex & $11(50.0)$ \\
Female & $11(50.0)$ \\
Male & \\
Time on project & $18(81)$ \\
Entire length of project & $4(18)$ \\
Partial length of project &
\end{tabular}

viduals with administrative support roles and student research assistants were not included in the sample because they lacked detailed knowledge of the project's scope and activities. Our research team worked closely with a physician research consulting firm, Alan Newman Research, for recruiting participants and facilitating interviews.

\section{Data Collection}

In-depth telephone and in-person interviews were conducted between January and May 2018 with members of the HVH research team. Experienced facilitators from Alan Newman Research conducted interviews and took summary notes, which lasted between 30 to 45 minutes. Each participant gave informed consent for the interview and audio recording, which were professionally transcribed for data analysis. All data collection efforts were directed by institutional review board-approved research protocols including a semistructured interview guide developed by our research team. Questions were designed to invoke analytic reflection and internal processing of specific phases and aspects of the project. Facilitators used "member checking" throughout the interview process by rephrasing key statements back to participants to verify accurate interpretation, which led to increased validity and credibility of the data. 


\section{Data Analysis}

Data analysis was performed by a multidisciplinary research team consisting of specialists in medicine, health care management, health economics, and qualitative and interpretive sociology. Three nonparticipant researchers who were not involved in the HVH initiative performed the qualitative analysis. One participant researcher, who was involved in the HVH initiative from the beginning of the project, provided contextual information on themes. NVivo version 11 was used for coding transcripts and analysis. Interview guides and summary notes were used to develop an initial set of codes, followed by refinement of codes based on themes emerging from the data. Descriptive codes were used in first cycle coding to assign labels to data, such as "challenges" or "worked well." We then used pattern coding to group first cycle codes into a smaller number of codes and then third cycle coding to finalize and organize key themes. Our research team met on a regular basis over an 8-month period for data analysis and kept detailed notes of coding comparisons, concept diagrams, and themes. To improve validity and credibility of findings, our research team elicited feedback from multiple study participants on key themes identified in the data analysis process. ${ }^{16}$

\section{Results}

This section is organized by research project phases to best present the key findings from $\mathrm{HVH}$ team member interviews. It is important to note that these phases occasionally overlapped. For example, tasks involved in evaluation, such as collecting and analyzing performance data, were performed throughout the project. A detailed listing of themes and corresponding quotes is provided in Table 2.

\section{Development}

Development of the initiative included HVH team member orientation and coordination, recruitment of practices, and development of tools and resources for practice improvement.

\section{Compressed Project Timeline}

The project timeline posed one of the largest challenges for the HVH initiative. HVH team members felt the period from grant award notification to the required intervention start was "rushed." The limited time made it difficult for HVH team mem- bers to complete project development tasks, which eventually compromised the original research design and shortened the intense intervention phase from 6 to 3 months. On reflection, most $\mathrm{HVH}$ team members believed the short project timeline was "completely unrealistic" and a precursor to other challenges.

\section{Recruitment Challenges}

The leadership team reported tremendous challenges recruiting 250 small-to-medium-sized practices, which they earlier thought possible because of their strong connections to the practice community. Recruitment was a challenge due to the short time frame and practice disinterest because of involvement with other government initiatives, particularly programs and requirements by the Centers for Medicare and Medicaid Services. Compromises, therefore, were made to the research design, including expanding the inclusion criteria to allow health system-owned practices to participate. Challenges were later experienced with many health system practices that were less engaged and less aware of the HVH initiative because health system leaders, not individual practice leaders, agreed to participate in the study. As $1 \mathrm{HVH}$ team member explained: "practices who were signed up by health systems it was harder to get them engaged because basically, someone outside of the practice... signed them up." In addition, according to interviewees, the hierarchical organizational structure of health system practices posed challenges because these practices did not have authority over many of their workflow processes, EHRs, and performance data.

\section{Collaborative Research Team}

One of the greatest strengths of the HVH initiative was the collaborative team of researchers, coaches, expert consultants, and evaluators. HVH team members thought of each other as "highly skilled," "excellent," and "knowledgeable." Members of the HVH team stated they "worked well together" and believed the project "benefited significantly from this not being a single institution project." HVH team members regularly held group meetings and referenced these meetings as pivotal for successful collaboration.

\section{Intervention Flexibility}

Intervention flexibility was a key component of research design, considering that participating practices were diverse in demographics, resources, 


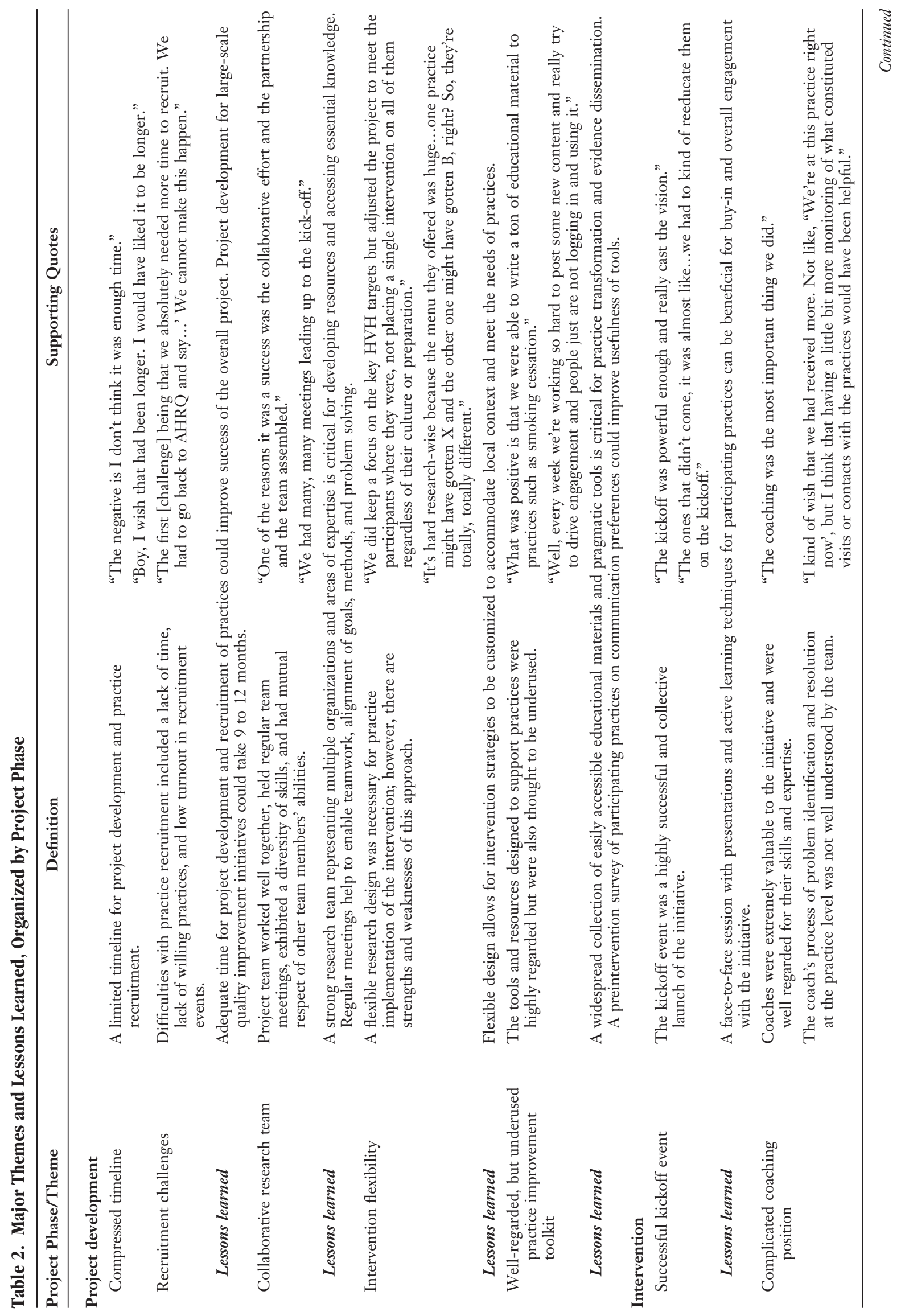




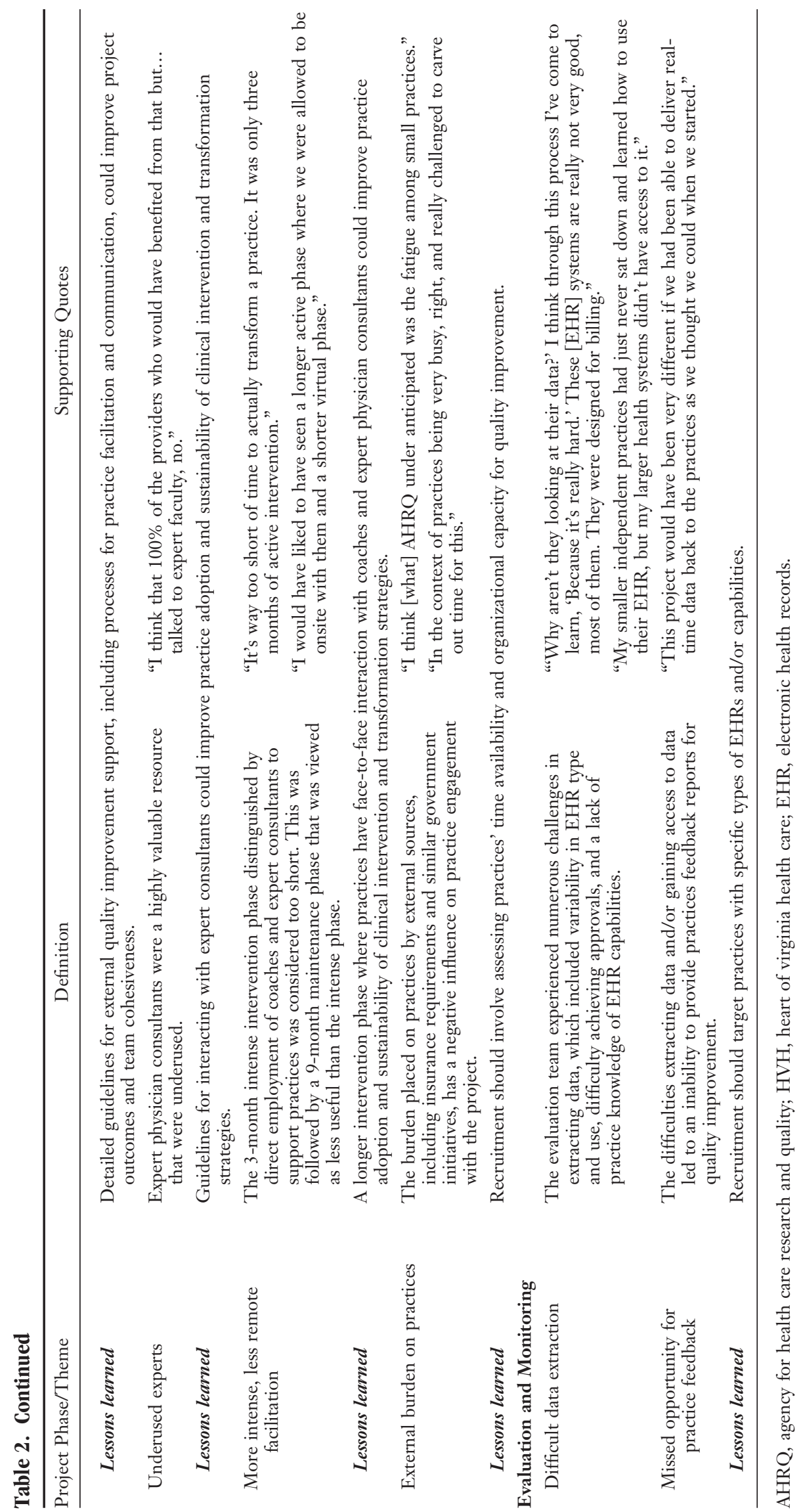


EHRs, and experience with data measurement. This flexibility allowed practices to select intervention strategies that best suited their needs and goals. Many $\mathrm{HVH}$ team members viewed this customized approach as positive, whereas evaluation team members desired a more standardized approach for outcome analysis. As 1 evaluation team member pointed out: "It is hard research-wise because the menu they offered was huge...1 practice might have gotten $X$, and the other 1 might have gotten B, right? So, they are totally, totally different." Several HVH team members suggested including a before and after assessment of practice transformation status and/or requiring that practice transformation activities serve as a prerequisite to a clinical intervention.

\section{Well-Designed Practice Improvement Toolkit}

$\mathrm{HVH}$ team members were highly pleased with the "toolkit" of resources developed for participating practices, which included checklists, educational/ training materials, and successful case studies on practice transformation and ABCS implementation. The research team, particularly the leadership team, exhibited great pride in the completed toolkit: "What was positive is that we were able to write a ton of educational materials to practices, such as smoking cessation." Still, despite its strengths, the research team recognized that the toolkit, as it was presented on the on-line platform was underused in the HVH initiative because people were "not logging in and using it.”

\section{Intervention}

The intervention phase of the research project was characterized by a kickoff event followed by interaction between $\mathrm{HVH}$ team members and participating practices. During the intense intervention phase, HVH coaches regularly met with practice staff to implement their customized list of strategies over a 3-month period, which was followed by a 9-month maintenance phase for practices to independently use $\mathrm{HVH}$ intervention strategies.

\section{Successful Kickoff Event}

The kickoff event was a highly positive and successful component of the HVH initiative. Interview participants described participation in the kickoff event as "critical" for initiating a practice's engagement and readiness and expressed viewpoints such as "practices that came and engaged that first day seemed much more likely to stay engaged." Practices that did not attend the kickoff were believed to be at a disadvantage and required additional orientation to the project. Multiple HVH team members suggested that the kickoff event be a mandatory component of participation in future large-scale quality improvement initiatives.

\section{Complicated Coaching Position}

The intense intervention phase was distinguished by direct employment of coaches and availability of expert consultants to participating practices. Both coaches and expert consultants were highly valued components of the initiative. Coaches were responsible for supporting practices in selecting and implementing their customized strategies. Coaches served as the face and facilitator of the HVH initiative for practices and in extreme cases coaches were considered a "lifeline" to "drowning practices." HVH team members had mixed feelings about the coaching organization's effectiveness in communicating challenges, concerns, and progress with practices. One HVH team member hesitated, then stated "the coaching organization...were not as great...as far as sharing what was going on." Reflecting on these challenges, numerous $\mathrm{HVH}$ team members pointed to factors such as the coaching organization's "closed culture," a lack of project "guidelines for coaching activities," and constraints of the coaching organization due to concurrent participation in other initiatives such as the Centers for Medicare and Medicaid Services Transforming Clinical Practice Initiative.

\section{Underused Experts}

Expert consultants, regarded as an extremely valuable resource, were "were hardly used" and seen as a "missed opportunity" to help practices with improvement efforts. Several factors were offered as to why expert consultants were underused, including a lack of role clarity, a perceived lack of time or availability, and a lack of involvement with coaches. Both coaches and expert consultants voiced a desire to have expert consultants more involved with practices during the intense intervention phase.

\section{More Intense, Less Remote Facilitation}

Multiple $\mathrm{HVH}$ team members, particularly the coaches, described the intense intervention phase as "too short" to "transform a practice." The lack of time in the intense intervention phase left many 
practices unprepared for the subsequent 9-month maintenance phase. The maintenance phase was designed for practices to independently continue implementing intervention strategies but was not a focal point of the initiative. Some coaches described this period as "hands off," which frequently resulted in no communication between the HVH coach and practice staff. Most coaches expressed a desire for a longer phase to work closely with practices and a concern that $\mathrm{HVH}$ virtual resources, such as on-line materials, were underused. Many $\mathrm{HVH}$ team members also voiced concern over the lack of project closure. Numerous coaches interviewed for this study shared a desire for a closing activity that would incorporate strategies for practices to move forward with the intervention after the HVH initiative ended.

\section{External Burden on Practices}

$\mathrm{HVH}$ team members unanimously agreed that it was difficult to achieve the HVH clinical intervention and practice transformation goals considering the external burden placed on practices. Many coaches also noted a challenge with external demands on practices: "I've got practices that are trying to manage 5 major insurances and trying to do proactive or quality improvement with all their patients for each insurance company. And Medicare, and MIPS, and Meaningful Use, and it is just too much."

\section{Evaluation}

\section{Difficult Data Extraction}

The HVH evaluation team worked closely with practices to obtain EvidenceNOW-required clinical outcomes data. However, the team experienced tremendous difficulty extracting data from practice EHRs. A member of the HVH evaluation team stated that getting data were a "6 to 12 -month lag, and for some (practices) we cannot get anything unless we physically go get it, and we should not do that more than 2 or 3 times." Most HVH leadership and evaluation team members indicated that they were not prepared for the challenges of working with a large number of practices with varying types and capabilities of EHRs. "I do not think any collaborative participating in this with AHRQ had any idea that that is how bad the state of reality versus what $\mathrm{ONC}$ was telling folks was. There was a huge disconnect."
Challenges in extracting data differed based on practice ownership, which were mainly due to bureaucracy in health system practices and a lack of trained personnel and expertise in independent practices. The challenges in extracting EHR data from practices ultimately suspended the plan to provide each practice with performance feedback reports.

\section{Missed Opportunity for Practice Feedback}

Coaches and other HVH team members voiced frustration in not obtaining performance feedback reports as originally planned in the $\mathrm{HVH}$ initiative. Many HVH team members referred to the situation as a "lost opportunity" for practice improvement. The team stated that a clear majority of the participating practices did not have access to their $\mathrm{HVH}$ performance data. Multiple team members commented that they would have liked to have practices to be able to successfully integrate evidence-based material into their practice in a sustainable way but that goal was not achieved. As 1 member of the HVH coaching team stated: "we could have done a little more actual QI [quality improvement] work if we had seen that data, but it just really was not feasible."

\section{Discussion}

Objectives of the HVH initiative were to disseminate research evidence in primary care practices in Virginia and generate information about the effectiveness of external quality improvement support. The initiative was designed as a pragmatic intervention to allow for local adaptation and flexibility. ${ }^{17}$ The goals of this study were to understand challenges and strengths of the project within local context. Major HVH challenges included recruiting 250 small-to-medium-sized primary care practices, extracting data from various types of EHRs, varying levels of practice engagement, working with practices at different stages of practice transformation and with different objectives for participating, and competing with other government initiatives. Strengths of the HVH initiative included a wealth of skills and expertise of HVH team members, regular research team meetings, well designed kick-off event and resources for practice improvement, and a flexible approach to meeting the needs of individual practices. 
The HVH initiative experienced challenges in the project development phase resulting in a domino effect that caused changes in the intervention phase, particularly the timeline and the length and intensity of onsite quality improvement assistance. Difficulty with EHR data extraction, ${ }^{18}$ which was experienced by other regional cooperatives, resulted in unintended consequences including changes in resource allocation and planned activities for quality improvement support. Some challenges may have been due to the diversity of practices in the study that included independent practices and health system-owned practices, practices at extreme ends of a practice transformation continuum, as well as varying types and capabilities of EHRs. Other challenges were related to the numerous goals of the EvidenceNOW initiative that required cooperatives to conduct a clinical intervention coupled with practice transformation.

There are multiple lessons learned in this study that could assist future efforts of implementation and dissemination research in primary care practices. Perhaps the key lesson learned was the need for additional time and resources for practice recruitment, ${ }^{19,20}$ a conclusion reached by other EvidenceNOW cooperatives. There is also a need for a longer intense intervention phase where practices have ongoing interaction with external quality improvement specialists, including physician consultants. Efforts should be taken, before the intervention, to assess antecedent organizational structures ${ }^{21}$ and processes for quality improvement such as EHR type, capability for performance measurement, and management approval processes for intervention-related changes. ${ }^{22,23}$ Efforts should be made to reach agreement among collaboration members on the priority of research goals and develop specific guidelines for external quality improvement support.

Practice transformation and adoption of evidence-based research require time commitments and major changes in processes, workflows, and training. This study supports previous reports from the EvidenceNOW initiative that cross-organizational collaboration requires extra time and resources to provide external quality improvement support to primary care practices to implement evidence-based research and conduct rigorous evaluations. ${ }^{17,24}$

Several limitations should be acknowledged when reviewing the results of this research. The first is that the experiences and viewpoints of study participants are only reflective of the $\mathrm{HVH}$ initiative and cannot be generalized to other EvidenceNOW regions or projects. There is also a possibility for recall bias among the practice coaches because most of these team members rolled off the project 6 to 12 months before the in-depth interviews and had difficulty remembering and responding to interview questions.

\section{Conclusion}

These study findings provide researchers, policy makers, and clinicians with insights for future quality improvement efforts, specifically the dissemination of evidence-based research, in primary care settings. The effectiveness of external quality improvement support may depend on practice engagement, antecedent quality improvement and organizational structures and processes, availability of practical tools and resources, and length and continuity of practice facilitation. Future large-scale primary care practice improvement efforts may benefit from a narrower focus on either clinical intervention or practice transformation and/or prerequisite organizational structures and processes before clinical intervention efforts.

To see this article online, please go to: http://jabfm.org/content/ 32/5/705.full.

\section{References}

1. Taylor ER, Peikes D, Genevro J, Meyers D. Creating capacity for improvement in primary care. Rockville MD: Agency for Healthcare Research and Quality. Available from: https://www.ahrq.gov/ professionals/prevention-chronic-care/improve/ capacity-building/pcmhqi1.html. Published 2013.

2. Centers for Disease Control and Prevention. National Center for Chronic Disease Prevention and Health Promotion, Division for Heart Disease and Stroke Prevention. Strategies for states to address the "ABCS" of heart disease and stroke prevention. Available from: https:/www.cdc.gov/dhdsp/programs/ spha/docs/abcs_guide.pdf. Published 2010.

3. Ono SS, Crabtree BF, Hemler JR, Balasubramanian BA. Taking innovation to scale in primary care practices: the functions of health care extension. Health Aff 2018;37:222-30.

4. Agency for Healthcare Research and Quality (AHRQ). Department of Health and Human Services. Accelerating the dissemination and implementation of PCOR findings into primary care practice (R18). Funding Opportunity Announcement (FOA) Number RFA-HS-14-008. Available from: https:// 
grants.nih.gov/grants/guide/rfa-files/RFA-HS-14008.html. Published 2014.

5. Ryan J, Doty MM, Hamel L, Norton M, Abrams MK, Brodie M. Primary care providers' views of recent trends in health care delivery and payment. The Commonwealth Fund Web site. Available from: https://www.commonwealthfund.org/publications/ issue-briefs/2015/aug/primary-care-providers-viewsrecent-trends-health-care-delivery. Published 2015.

6. Goldberg DG, Mick SS, Kuzel AJ, Feng LB. Love LE. Why do some primary care practices engage in practice improvement efforts whereas others do not? Health Serv Res 2013;48:398-416.

7. Longo DR, Rothemich SF, Krist AH, et al. Quality performance monitoring, data collection, and reporting. Report of experiences from primary care practices in the Virginia Ambulatory Care Outcomes Network. HHSA 290-200710011-2. Available from: https:/healthit.ahrq.gov/sites/default/files/docs/ citation/quality-performance-monitoring-datacollection-and-reporting-final-report.pdf. Published 2015.

8. Kane CK. Updated data on physician practice arrangements: physician ownership drops below 50 percent. AMA policy research perspectives. AMA Web site. Available from: https://www.ama-assn. org/sites/ama-assn.org/files/corp/media-browser/ public/health-policy/PRP-2016-physician-benchmarksurvey.pdf. Published 2016.

9. Cuellar A, Krist AH, Nichols LM, Kuzel AJ. Effect of practice ownership on work environment, learning culture, psychological safety, and burnout. Ann Fam Med 2018;16:S44-S51.

10. Holtrop JS, Rabin BA, Glasgow RE. Dissemination and implementation science in primary care research and practice: contributions and opportunities. J Am Board Fam Med 2018;31:466-78.

11. Nguyen QD, Fernandez N, Karsenti T, Charlin B. What is reflection? A conceptual analysis of major definitions and a proposal of a five-component model. Med Educ 2014;48:1176-89.

12. Lanham HJ, Palmer RF, Leykum LK, et al. Trust and reflection in primary care practice redesign. Health Serv Res 2016;51:1489-514.
13. Lutz G, Roling G, Berger B, Edelhäuser F, Scheffer C. Reflective practice and its role in facilitating creative responses to dilemmas within clinical communication-a qualitative analysis. BMC Med Educ 2016;16:301.

14. Sanders J. The use of reflection in medical education. Med Educ 2009;31:408-15.

15. Veno M, Silk H, Savageau J, Sullivan K. Evaluating our strategy for including reflection in medical education and practice. Fam Med 2016;48:300-4.

16. Lincoln YS, Guba EG. Naturalistic inquiry. Newberry Park (CA): Sage Publications, Inc.; 1985.

17. Meyers D, Miller T, Genevro J, et al. EvidenceNOW: balancing primary care implementation and implementation research. Ann Fam Med 2018;16: S5-S11.

18. Cohen DJ, Dorr DA, Knierim K, et al. Primary care practices' abilities and challenges in using electronic health record data for quality improvement. Health Aff 2018;37:635-43.

19. Sweeney SM, Hall JD, Ono SS, et al. Recruiting practices for change initiatives is hard: findings from EvidenceNOW. Am J Med Qual 2018;33:1-7.

20. Fagnan LJ, Walunas TL, Parchman ML, et al. Engaging primary care practices in studies of improvement: did you budget enough for practice recruitment? Ann Fam Med 2018;16:S72-S9.

21. Harrison M, Kimani J. Building capacity for a transformation initiative: System redesign at Denver Health. Health Care Manage Rev 2009;34:42-53.

22. Balasubramanian BA, Marino M, Cohen DJ, et al. Use of quality improvement strategies among small to medium-size US primary care practices. Ann Fam Med 2018;16:S35-S43.

23. Kaplan HC, Brady PW, Dritz MC, et al. The influence of context on quality improvement success in health care: a systematic review of the literature. Milbank Q 2010;88:500-59.

24. Hemler JR, Hall JD, Cholan RA, et al. Practice facilitator strategies for addressing electronic health record data challenges for quality improvement: EvidenceNOW. J Am Board Fam Med 2018;31:398409 . 